\title{
AVALIAÇÃO FÍSICA E MECÂNICA DE MÁRMORE ARTIFICIAL PRODUZIDO COM RESÍDUO FINO DE MÁRMORE DOLOMÍTICO BENEFICIADO EM TEARES DE LÂMINAS DIAMANTADAS*
}

Tcharllis João da Cunha Demartini ${ }^{1}$ Rubén Jesus Sánchez Rodriguez ${ }^{2}$

\section{Resumo}

A produção de uma rocha artificial a partir da aglutinação de resíduos finos de mármore dolomítico pela resina epóxi pode ser uma alternativa viável do ponto de vista técnico, econômico e ecológico. O resíduo utilizado é proveniente do desdobramento de mármore dolomítico em teares de lâminas diamantadas e geralmente é descartado. Esta nova aplicação agrega valor ao resíduo, diminuindo o impacto ambiental gerado. $O$ objetivo deste trabalho foi avaliar as propriedades físicas e mecânicas do mármore artificial produzido pela metodologia de vibro compressão à vácuo. Para isso, foram confeccionadas placas rochosas, em equipamento apropriado, com $85 \%$ em peso de partículas de resíduo e $15 \%$ de sistema epoxídico DGEBA/TEPAc. As placas foram produzidas com uma pressão de compactação de $0,5 \mathrm{MPa}$, à temperatura de $90{ }^{\circ} \mathrm{C}$ por um período de 20 minutos. Posteriormente, as placas foram caracterizadas pelos ensaios de densidade, absorção de água, porosidade, resistência à flexão, resistência à compressão e microscopia eletrônica de varredura. Obteve-se um mármore artificial produzido pela técnica de vibro compressão a vácuo com absorção de água inferior a 0,06\%, uma resistência à flexão de 33,93MPa e uma compressão uniaxial de $96,49 \mathrm{MPa}$, confirmando uma boa adesão entre as partículas de resíduo e a resina epóxi.

Palavras-chave: Mármore artificial; Resíduo; Vibro compressão a vácuo; Resina epóxi.

\section{PHYSICAL AND MECHANICAL EVALUATION OF ARTIFICIAL MARBLE PRODUCED WITH DOLOMITIC MARBLE WASTE BENEFITED BY LOOMS OF DIAMOND BLADES}

\section{Abstract}

The production of artificial stone from the agglutination of the epoxy resin fine waste can be a viable alternative from technical, economic and ecological point of views. The residue derives from the dolomitic marble unfolding into looms of diamond blades and it is usually discarded. With this new application, there is value added to the residue, reducing the consequent environmental impact. The main objective of this research is to evaluate the physical and mechanical properties of an artificial marble produced through vacuum vibro-compression. With this purpose, stone plates were produced, using appropriate tools, characterized by a distribution of $85 \%$ of residual particles weight and $15 \%$ of epoxy system DGEBA/TEPAc. The products were manufactured with the following conditions during 20 (twenty) minutes, compacting pressure of $0.5 \mathrm{MPa}$ and temperature of $90{ }^{\circ} \mathrm{C}$. Later on, the samples were characterized by density, water absorption, porosity, flexural strength, compressive strength and scanning electron microscopy. The result was an artificial marble produced employing vacuum vibrocompression with water absorption levels below $0.06 \%$, flexural strength of $33.93 \mathrm{MPa}$ and uniaxial compression $96.49 \mathrm{MPa}$, thus indicating residual particles and epoxy resin grip satisfactory levels.

Keywords: Artificial stone; Residue; Vacuum vibro-compression; Epoxy resin.

Pós-graduação em Engenharia e Ciência dos Materiais, M.Sc., LAMAV, Universidade Estadual do Norte Fluminense Darcy Ribeiro - UENF, Campos dos Goytacazes, Rio de Janeiro, Brasil.

2 Pós-graduação em Ciências Químicas (Polímeros), D.Sc., Professor, LAMAV, Universidade Estadual do Norte Fluminense Darcy Ribeiro - UENF, Campos dos Goytacazes, Rio de Janeiro, Brasil. 


\section{INTRODUÇÃO}

O Brasil é o quarto maior produtor mundial de rochas ornamentais com uma produção estimada de 9,5 milhões de toneladas [1].

Durante a produção de rochas ornamentais são gerados resíduos principalmente durante os processos de extração e desdobramento de blocos de rochas em chapas, que necessitam de maiores áreas para disposição diariamente [2].

Como causam danos ao meio ambiente, os resíduos de rochas, apesar de na maioria das vezes serem descartados, poderiam ser reciclados e reutilizados de forma sustentável através da implementação de projetos baseados em pesquisas acadêmicas.

Uma alternativa para a reciclagem dos resíduos provenientes do desdobramento de rochas ornamentais em equipamentos diamantados proposta nesta pesquisa é a sua aglutinação através de resina epóxi pelo método de vibro-compressão a vácuo, desenvolvendo-se rochas artificiais, como por exemplo o mármore artificial compacto.

O mármore artificial é bem aceito pelo mercado consumidor, sendo várias as vantagens citadas pelas empresas que o produzem e comercializam. A principal delas é a impermeabilidade, impedindo a ocorrência de manchamento, atribuída à aplicação de resina em sua constituição, que une as partículas de rocha e preenche a porosidade típica de rochas naturais. Assim, não há penetração e percolação de líquidos em seu interior [3,4].

No Brasil e no mundo, ao longo dos últimos anos, a demanda é crescente por rochas artificiais $[1,5]$. Somente entre os anos de 2011 e 2016, foi estimado que haveria um aumento de $36 \%$ no seu consumo [5].

Diante deste contexto, fica evidente que a reciclagem de resíduos na produção de rochas ornamentais é uma alternativa viável tanto do ponto de vista técnico quanto ecológico, além de agregar valor a algo que, com menor ou maior grau de adequação as leis ambientais, seria descartado no meio ambiente. Essa iniciativa ainda pode contribuir para o desenvolvimento das atuais tecnologias de produção de rochas artificiais e aumento dos postos de trabalho no setor.

\section{MATERIAIS E MÉTODOS}

Foram utilizados nesta pesquisa dois tipos de resíduos originados no desdobramento de mármore dolomítico natural, comercialmente denominado de "Marmo-Onyx Blue", originário da empresa Santa Clara, localizada no distrito de Gironda em Cachoeiro de Itapemirim, ES, Brasil.

O primeiro resíduo é o semissólido e corresponde ao material coletado diretamente na empresa, no sistema de aspersão de lama abrasiva, denominado chuveiro, do tear de lâminas diamantadas do setor de beneficiamento. Após a coleta, este resíduo foi submetido à estufa, para retirada da umidade. O segundo resíduo é o sólido e corresponde aos casqueiros eliminados durante o desdobramento do mármore no mesmo tear, eles foram britados em um britador de mandíbulas, moídos em um moinho de discos de porcelana e peneirados a seco em um peneirador vibratório de bancadas em duas faixas granulométricas, grossa $(2,000-0,425 \mathrm{~mm})$ e média $(0,425-0,075 \mathrm{~mm})$. A lama seca também foi peneirada e separada na faixa granulométrica fina. $(<0,075 \mathrm{~mm})$. 
Esta distribuição do tamanho de partículas foi realizada pelo método de peneiramento fino, de acordo com a norma técnica ABNT NBR 7181, utilizando peneiras entre 10 e 200 mesh [6].

A partir das três faixas granulométricas foram determinadas 10 misturas com porcentagens distintas de partículas grossas, médias e finas. Para isso utilizou-se um diagrama ternário de modelo cúbico completo, que foi desenvolvido na metodologia de modelagem numérico experimental rede Simplex (Simplex-Lattice Design) [7]. O objetivo principal foi determinar qual destas misturas apresentou a densidade aparente seca máxima. O ensaio foi baseado na norma brasileira ABNT MB-3388 [8] e a mistura com maior densidade aparente seca obtida representa 0 maior empacotamento das partículas (4/6 para partículas grossas, 1/6 de médias e $1 / 6$ de finas), a qual foi escolhida para a produção das placas de rocha artificial.

As partículas do resíduo de mármore foram aglutinadas pela resina epóxi do tipo diglicidil éter de bisfenol A (DGEBA) endurecida por uma amina comercial composta por aproximadamente $60 \%$ de Tetraetilenopentamina (TEPA) e $40 \%$ de Trietilenotetramina (TETA) com algumas impurezas. Foi calculado um teor mínimo de resina a ser utilizado para preencher a porosidade entre as partículas e por isso foi utilizada uma mistura de $85 \%$ de partículas e $15 \%$ de resina epóxi.

As placas rochosas foram confeccionadas nas dimensões de $200 \times 200 \times 10 \mathrm{~mm}$ pela metodologia de vibro-compressão a vácuo. A mistura foi submetida a vácuo ao mesmo tempo em que se aplicava uma pressão de compactação de $0,5 \mathrm{MPa}$ a $90^{\circ} \mathrm{C}$ durante 20 minutos objetivando a sua cura.

Posteriormente, as placas foram cortadas nas dimensões específicas de ensaios que visaram a sua caracterização.

Os valores de densidade, absorção de água e porosidade aparentes foram obtidos pelo ensaio de índices físicos regulamentado pela norma ABNT NBR 15845- anexo B [9].

Foram confeccionados 10 corpos de prova com dimensões $30 \times 30 \times 30 \mathrm{~mm}$, os quais foram secos em estufa ventilada com temperatura de $70 \pm 5^{\circ} \mathrm{C}$, até atingir a massa seca constante aferida pela pesagem em balança de precisão.

Para a determinação da massa saturada, os corpos de prova foram colocados em uma bandeja de aço inoxidável e imersos em água destilada ou deionizada, inicialmente a 1/3 da altura dos corpos de prova, e após 4 h até $2 / 3$ da altura. Após esse período completou-se a submersão da altura total por mais $40 \mathrm{~h}$ e ao fim deste tempo foram pesados.

A última etapa consistiu na pesagem dos corpos quando são imersos em recipiente com água potável através de um fio de nylon preso sob a balança ou utilizando um dispositivo de pesagem hidrostática. O peso de cada corpo de prova nestas condições é chamado de peso submerso.

Para definir os valores das três propriedades foram utilizadas as equações 1,2 e 3 .

$$
\begin{array}{r}
\rho_{a}=M_{s e c}\left(M_{s a t}-M_{s u b}\right) \times 1000 \rho_{a}=M_{s e c}\left(M_{s a t}-M_{s u b}\right) \times 1000(1) \\
n_{a}=\left(M_{s a t}-M_{s e c}\right)\left(M_{s a t}-M_{s u b}\right) \times 100 n_{a}=\left(M_{s a t}-M_{s e c}\right)\left(M_{s a t}-M_{s u b}\right) \times 100(2) \\
\alpha_{a}=\left(M_{s a t}-M_{s e c}\right) M_{s e c} \times 100 \alpha_{a}=\left(M_{s a t}-M_{s e c}\right) M_{s e c} \times 100
\end{array}
$$

Para a realização do ensaio de resistência à flexão em três pontos foi utilizada a máquina universal de ensaios EMIC modelo DL10000 O ensaio é normatizado pela NBR 15845/2010-Anexo F [9] e UNE-EN 14617-2:2008 [10]. Ao todo foram utilizados 6 corpos com dimensões de $10 \times 25 \times 100 \mathrm{~mm}$. Ao fim do ensaio foram calculados a tensão máxima de ruptura e o desvio padrão. 
O ensaio de resistência à compressão se baseou nas diretrizes estabelecidas pelas normas NBR 15845/2010- Anexo E [9], brasileira, e espanhola UNE-EN 14617-15 [11] e foi realizado na máquina universal de ensaios EMIC modelo DL10000. Foram utilizados 10 corpos de prova secos em estufa conforme a norma e com dimensões

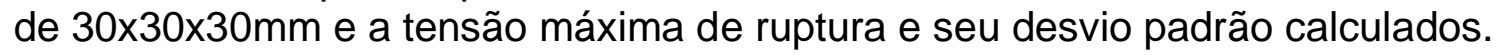

Além do mármore artificial produzido, também foi caracterizado o mármore artificial comercial Bianco Prime fornecido pela Emporio Stone nos testes mecânicos (flexão e compressão) para posterior comparação dos resultados.

Avaliou-se a microestrutura da região de fratura dos corpos de prova submetidos ao ensaio de flexão por um Microscópio Eletrônico de Varredura (MEV), possibilitando analisar a adesão das partículas ao sistema DEGBA-TEPAc. O equipamento utilizado para realizar estas análises foi o TMT3030PLUS fabricado pela HITACHI. As amostras foram preparadas previamente através do uso de fita adesiva de carbono envolvida com uma superfície de ouro.

\section{RESULTADOS E DISCUSSÃO}

São apresentados na tabela 1, os resultados dos índices físicos encontrados para o mármore artificial produzido através do método de Vibro compressão à vácuo, suas respectivas médias e desvios padrões.

Tabela 1. Resultados dos Índices Físicos para o Mármore Artificial Produzido

\begin{tabular}{lccc}
\hline $\begin{array}{c}\text { Corpo de } \\
\text { Prova }\end{array}$ & Densidade $\left(\mathbf{g} / \mathbf{c m}^{3}\right)$ & Porosidade (\%) & Absorção de Água (\%) \\
\hline $\mathbf{1}$ & 2,21 & 0,15 & 0,07 \\
\hline $\mathbf{2}$ & 2,07 & 0,12 & 0,06 \\
\hline $\mathbf{3}$ & 2,19 & 0,12 & 0,05 \\
\hline $\mathbf{4}$ & 2,03 & 0,15 & 0,07 \\
\hline $\mathbf{5}$ & 2,11 & 0,12 & 0,06 \\
\hline $\mathbf{6}$ & 2,11 & 0,12 & 0,08 \\
\hline $\mathbf{7}$ & 2,05 & 0,16 & 0,06 \\
\hline $\mathbf{8}$ & 2,11 & 0,12 & 0,07 \\
\hline $\mathbf{9}$ & 2,08 & 0,15 & 0,06 \\
\hline $\mathbf{1 0}$ & 2,08 & 0,12 & 0,06 \\
\hline Média & $\mathbf{2 , 1 0 \pm 0 , 0 6}$ & $\mathbf{0 , 1 3} \pm \mathbf{0 , 0 2}$ & $\mathbf{0 , 0 6} \pm \mathbf{0 , 0 1}$ \\
\hline
\end{tabular}

Para a densidade aparente foi encontrado o valor médio de $2,10 \pm 0,06 \mathrm{~g} / \mathrm{cm}^{3}$, que é aproximadamente $15 \%$ inferior aos valores informados pelas empresas que produzem rochas artificiais. Esses valores geralmente variam entre 2,4 a $2,5 \mathrm{~g} / \mathrm{cm}^{3}$ [12]. A densidade inferior reflete diretamente no peso por metro quadrado dos ladrilhos a serem fabricados a partir da rocha artificial produzida, então um material com mesmas dimensões será mais leve, o que diminui os custos de transporte. 
Lee et. al. [13] encontrou valores que variam entre 2,03 a 2,45 g/ $/ \mathrm{cm}^{3}$, para rochas artificiais produzidas sob a variação da pressão de compressão, frequência de vibração e nível de vácuo, ou seja, o mármore artificial produzido neste estudo possui densidade que está dentro dos valores encontrados pelo autor.

Quanto a porosidade aparente, o valor encontrado foi de $0,13 \pm 0,02 \%$, o que pode evidenciar uma adesão satisfatória entre as partículas e a resina.

Chiodi e Rodriguez [14] classificaram materiais destinados ao revestimento da construção civil e determinaram que eles têm alta qualidade quando possuem valores de porosidade inferiores a $0,5 \%$. A porosidade aparente encontrada para 0 mármore artificial produzido é aproximadamente quatro vezes menor que 0 determinado pelos autores.

Em se tratando da absorção de água do mármore artificial produzido foi determinado o valor de $0,06 \pm 0,01 \%$. O valor encontrado é inferior ao recomendado para mármore dolomítico natural que tem como indicação possuir um valor menor ou igual $0,20 \%$ [15].

Chiodi e Rodriguez [14] estabeleceram que um valor inferior a $0,1 \%$ atesta que a rocha possui uma qualidade muito alta, sendo o valor encontrado $40 \%$ inferior a esta classificação.

Mármores artificiais produzidos pela indústria possuem entre 0,09 a $0,40 \%$ de absorção de água [12]. Carvalho et. al. [16] encontrou o valor de 0,17\% de absorção de água. O mármore artificial produzido nesta pesquisa possui absorção de água inferior em aproximadamente $33 \%$ em relação ao mínimo da faixa de valores informados pela indústria e quase três vezes inferior ao encontrado por Carvalho et. al. [16]. Com estas comparações comprova-se a baixa absorção de água do material para a sua utilização em ambientes úmidos.

$\mathrm{Na}$ tabela 2 estão descritos os valores encontrados para a resistência à flexão em 3 pontos dos 6 corpos de prova confeccionados a partir do mármore artificial desenvolvido com $85 \%$ de resíduo de mármore dolomítico e $15 \%$ do sistema epoxídico DGEBA/TEPAc.

Tabela 2. Resultados da Resistência à Flexão em 3 Pontos do Mármore Artificial Produzido

\begin{tabular}{lc}
\hline Corpo de Prova & $\begin{array}{c}\text { Resistência à Flexão em 3 } \\
\text { Pontos (MPa) }\end{array}$ \\
\hline $\mathbf{1}$ & 34,05 \\
\hline $\mathbf{2}$ & 33,64 \\
\hline $\mathbf{3}$ & 33,95 \\
\hline $\mathbf{4}$ & 33,42 \\
\hline $\mathbf{5}$ & 34,83 \\
\hline $\mathbf{6}$ & 33,72 \\
\hline Média & $\mathbf{3 3 , 9 3} \pm \mathbf{0 , 4 9}$ \\
\hline
\end{tabular}

O desvio padrão de $0,49 \mathrm{MPa}$ encontrado demonstra uma faixa bastante reduzida da variação no comportamento mecânico do material neste teste, permitindo inferir que as placas da rocha artificial produzida utilizadas neste teste eram bem homogêneas na sua constituição, o que pode ser reflexo de um entrecruzamento eficiente das 
cadeias poliméricas durante a cura da resina e sua boa adesão às partículas do resíduo por toda a superfície rochosa.

Os fabricantes de rocha artificial produzidas com partículas de mármore fornecem valores de resistência à flexão em torno de 13,6 a 17,2MPa [17]. O valor encontrado nesta pesquisa é quase o dobro do máximo informado para um mármore artificial comercial desenvolvido por estas empresas.

Borsellino et al. [18]. utilizou partículas de mármore e as aglutinou com resina epóxi, sem utilização da metodologia de vibro compressão a vácuo, obtendo valores de resistência à flexão entre 10,6 e 22,2MPa. O valor de resistência encontrado para o mármore artificial produzido é $53 \%$ superior ao máximo encontrado pelo autor. Infere-se que à utilização da técnica de vibro compressão a vácuo durante a confecção do mármore artificial desta pesquisa diminuiu os níveis de porosidade, impedindo a formação de concentradores de tensão.

O módulo de elasticidade encontrado foi de 7,10 $\pm 0,6 \mathrm{GPa}$, indicando que 0 mármore artificial produzido tem elevada rigidez durante a flexão. A boa adesão interfacial entre as partículas de mármore dolomítico e sua dispersão homogênea na resina epóxi, com bom molhamento, beneficiou o incremento do módulo de elasticidade, sendo ele superior aos obtidos para o sistema epoxídico e o que geralmente é definido para mármores naturais.

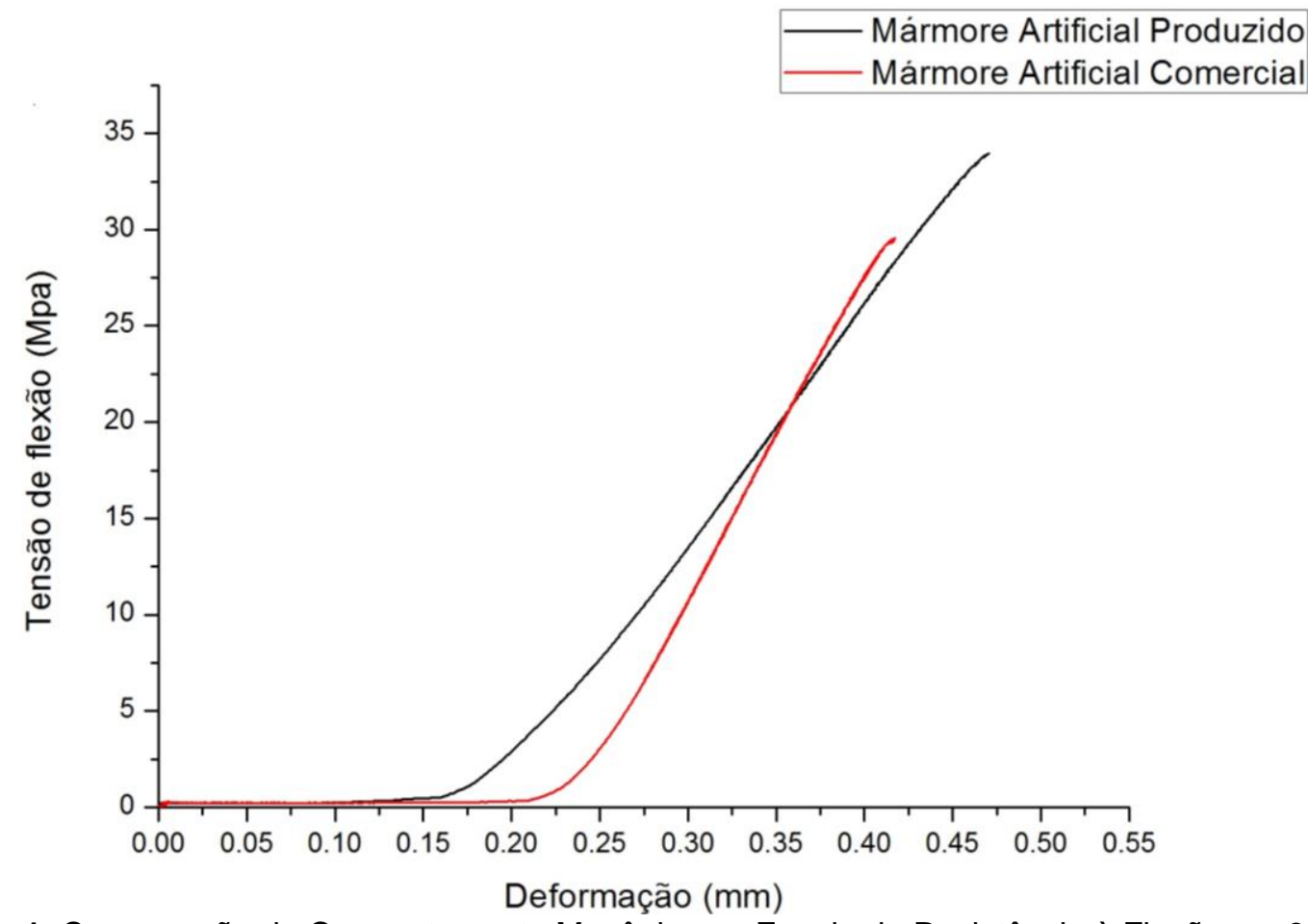

Figura 1. Comparação do Comportamento Mecânico no Ensaio de Resistência à Flexão em 3 Pontos entre o Mármore Artificial Produzido e o Mármore Artificial Comercial

Na figura 1 é possível estabelecer uma comparação entre os comportamentos mecânicos do mármore artificial produzido e do mármore artificial comercial. Para o mármore artificial comercial foi obtida a tensão máxima de ruptura de 30,42 \pm 2,37MPa, demonstrando que o mármore artificial produzido possui uma resistência à flexão em três pontos superior em quase 12\%. Isso demonstra que o material produzido está em conformidade com o que se espera em termos de resistência por um semelhante produzido pela indústria.

$\mathrm{Na}$ Tabela 3 estão descritos os resultados obtidos no ensaio de resistência à compressão uniaxial. 
Tabela 3. Resistência à Compressão Uniaxial do Mármore Artificial Produzido

\begin{tabular}{lc}
\multicolumn{1}{c}{ Corpo de Prova } & $\begin{array}{c}\text { Resistência à Compressão } \\
\text { Uniaxial (MPa) }\end{array}$ \\
\hline $\mathbf{1}$ & 93,13 \\
\hline $\mathbf{2}$ & 96,39 \\
\hline $\mathbf{3}$ & 95,81 \\
\hline $\mathbf{4}$ & 100,95 \\
\hline $\mathbf{5}$ & 96,19 \\
\hline Média & $\mathbf{9 6 , 4 9 \pm \mathbf { 2 , 8 2 }}$ \\
\hline
\end{tabular}

Foi obtido o valor de $96,49 \pm 2,82 \mathrm{MPa}$ no teste de resistência à compressão. Este resultado evidencia que houve uma boa interligação das cadeias poliméricas da resina, reduzindo a os escorregamentos nos planos de fraqueza que comumente ocorrem nos mármores artificiais quando submetidos a esforços compressivos.

Quando este valor é comparado à regulamentação definida pela norma ASTM C503 para a matéria prima que são os mármores dolomíticos naturais, ele se prova superior, sendo quase o dobro do valor mínimo estabelecido pela norma que é de $52 \mathrm{MPa}[15]$.

Lee et al. [13], utilizando partículas graníticas e vítreas que possuem resistência mecânica mais elevada que as dolomíticas deste estudo, variou os parâmetros de fabricação do mármore artificial e obteve valores na faixa de 78,7 até 151,3MPa.

Para efeitos de classificação, uma vez que não existem normas próprias para isto no campo das rochas artificiais, utilizaram-se os valores definidos por Chiodi e Rodriguez [14]. Os autores definem que quando utilizadas para fins estruturais, as rochas artificiais devem apresentar valores de resistência à compressão situados entre 70 e 130MPa para uma resistência média, classificação que engloba o mármore artificial produzido nesta categoria.

$\mathrm{Na}$ comparação entre o mármore comercial Bianco Prime e o mármore artificial produzido, o último obteve como resultado quase o dobro de resistência do primeiro, $96,49 \pm 2,82 \mathrm{MPa}$ contra 56,46 $\pm 14,46 \mathrm{MPa}$, conforme visto na figura 2 . 


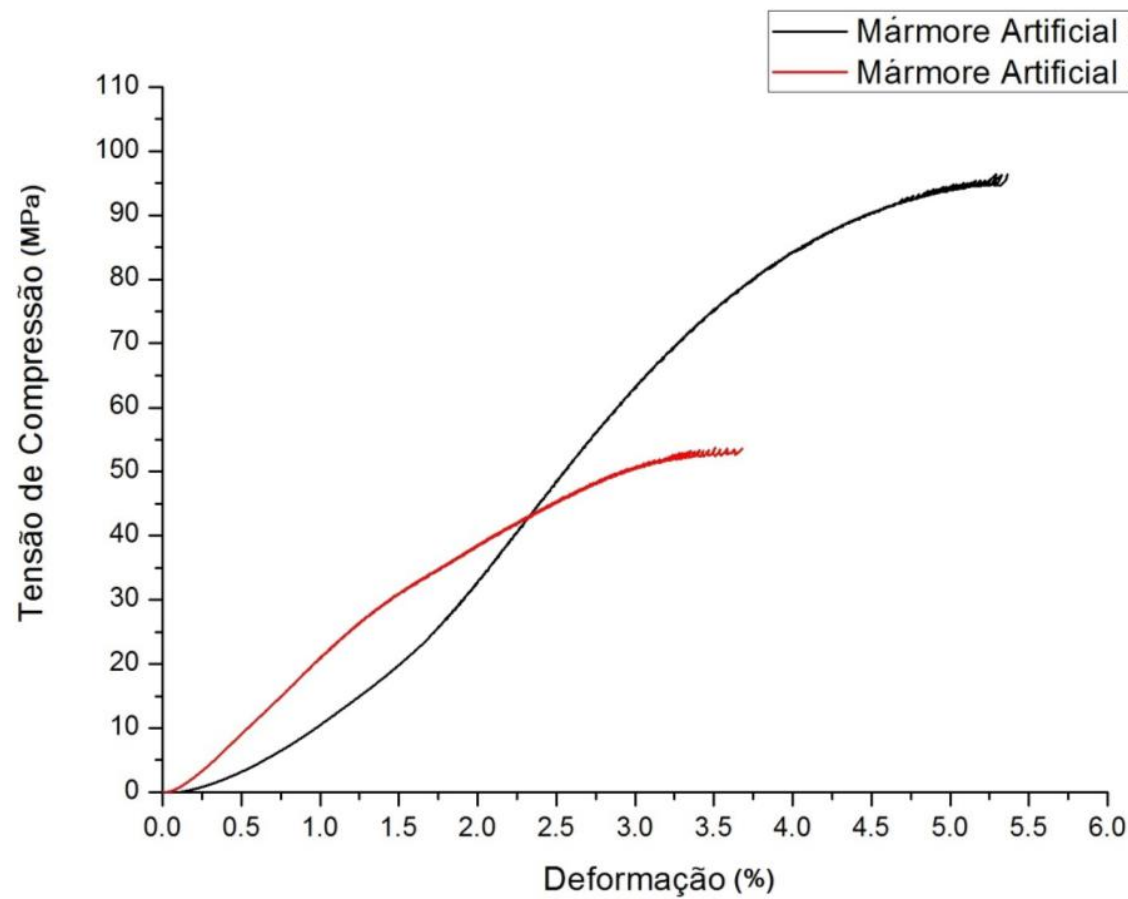

Figura 2. Comparação entre o Comportamento Mecânico na Compressão do Mármore Artificial Produzido X Mármore Artificial Comercial

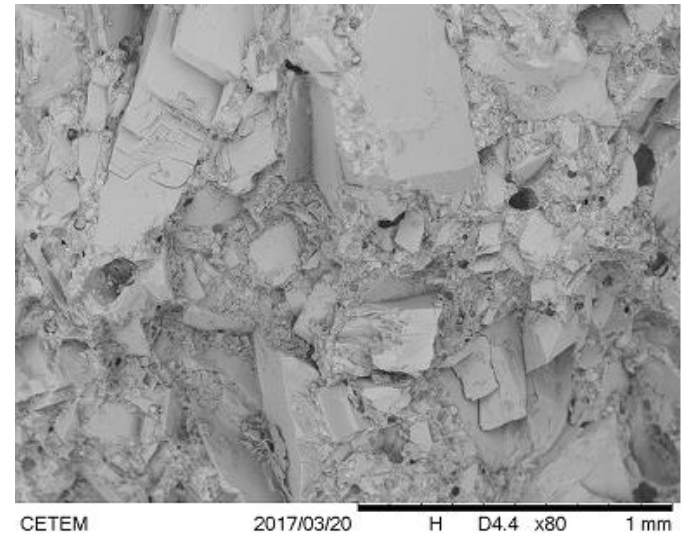

a

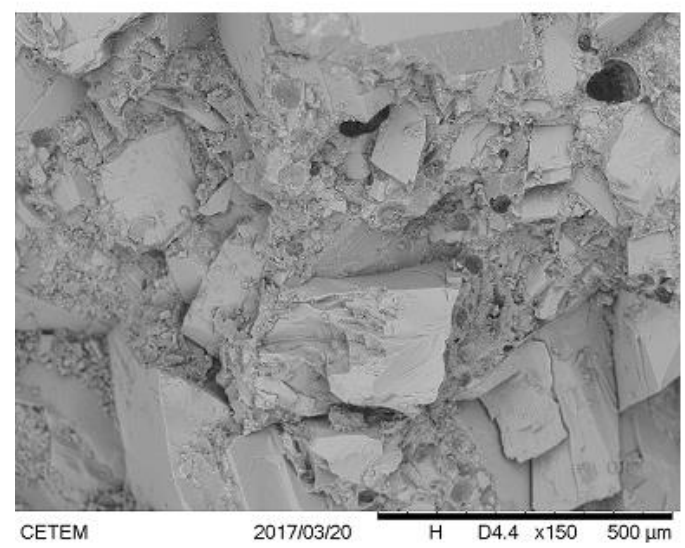

C

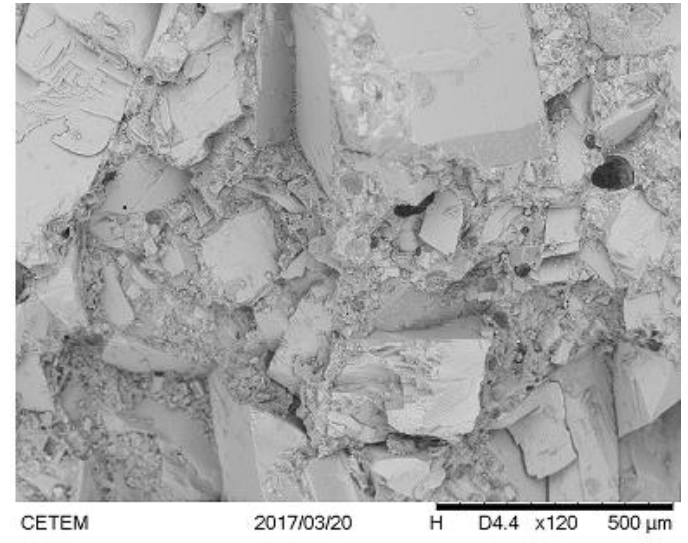

b

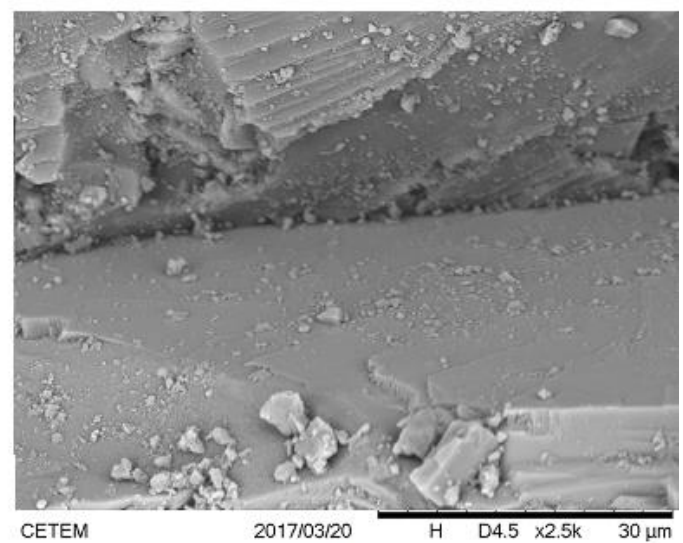

d

Figura 3. Microscopia Eletrônica de Varredura da Seção de Fratura do Mármore Artificial Produzido 
Na figura 3 (a, b, c, d) estão representadas as análises microscópicas obtidas por MEV realizado sobre a superfície de fratura obtida no ensaio de resistência à flexão em 3 pontos do mármore artificial produzido.

São poucos os poros evidentes, pois como comprovado pelos índices físicos a porosidade é bastante reduzida. No entanto, eles podem ter contribuído para a ocorrência da fratura, uma vez que se observa uma boa adesão interfacial entre partículas e resina, ou seja, ocorreu um molhamento adequado das partículas pela resina.

Miller et al. [19] e Debnath et al. [20] relataram que boas interações na interface do compósito podem melhorar as propriedades mecânicas dos compósitos, estando esta interação diretamente ligada à força de ligação adesiva proporcionada pelo molhamento eficaz nas regiões interfaciais.

Luz e Lins [21] descrevem a dolomita como um mineral com sistema cristalino hexagonal, comumente apresentando cristais romboédricos com faces curvadas.

A fratura intragranular ocorreu principalmente nos planos de clivagem da dolomita, pois apresentam superfícies com formatos romboédricos. Este faturamento ocorreu devido ao esforço mecânico empregado nestes planos preferenciais de fraqueza. $\mathrm{Na}$ região interfacial, a boa adesão permitiu como visto nos testes de flexão e compressão, resultados satisfatórios para estas propriedades.

Infere-se que a compactação prevista no diagrama ternário resultou em uma boa aderência nas regiões interfaciais e poucas falhas na superfície, resultando na baixa porosidade encontrada, o que aumenta a taxa de brilho quando o material passar por processos de beneficiamento como polimento de superfície e bordas. Tais fatores criam uma superfície menos penetrável por líquidos, dificultando a formação de manchas e melhoram as propriedades mecânicas, que como já visto, foram superiores ao do mármore artificial analisado.

\section{CONCLUSÃO}

Foi constatado através da caracterização do mármore artificial produzido pela metodologia de vibro-compressão a vácuo, com uma pressão de compactação de $0,5 \mathrm{MPa}$, a uma temperatura de $90^{\circ} \mathrm{C}$, por um período de 20 minutos e constituído por $85 \%$ em peso de resíduo de mármore dolomítico desdobrado em teares de lâminas diamantadas e 15\% do sistema epoxídico DGEBA/TEPAc, que:

- $\quad$ Foi produzido um mármore artificial compacto com excelentes propriedades físicas e mecânicas. Ele possui apenas $0,13 \%$ e $0,06 \%$ de porosidade e absorção de água, respectivamente, evidenciando sua alta impermeabilidade. Possui tensão máxima de ruptura na flexão de 33,93MPa e 96,49MPa na compressão, superiores ao de um mármore artificial comercial semelhante, demonstrando sua alta resistência mecânica.

- $\quad$ No MEV, as regiões de fratura demonstraram que as partículas dolomíticas aderiram bem ao sistema epoxídico DGEBA/TEPAc, com poucos poros visíveis, o que foi comprovado nos testes mecânicos, ressaltando o quanto foi importante definir a melhor mistura de granulometrias e escolher 0 maior fator de empacotamento pelo diagrama ternário.

Desta maneira, a aglutinação das partículas de resíduo de mármore dolomítico desdobrado em teares de lâminas diamantadas pelo sistema epoxídico DGEBA/TEPAc permite a confecção de um mármore artificial compacto sustentável com excelentes propriedades físicas e mecânicas. 


\section{Agradecimentos}

Os autores agradecem a FAPERJ e CNPq pelo apoio financeiro.

\section{REFERÊNCIAS}

1 Abirochas. Balanço das exportações e importações brasileiras de rochas ornamentais em 2015. 2016 [acesso em 22 mai. 2016];01:01-19. Disponível em: http://www.ivolution.com.br/mais/fotos/6/17/3397/Informe_01_2015.pdf.

2 Souza LR, Ribeiro RCC, Carrisso RCC, Silva LP, Pacheco EBAV, Visconte LLY. Aplicação de resíduos de mármore na indústria polimérica. Rio de Janeiro: CETEM/MCT, 2009.

3 Breton Company. Plants for manufaturing natural stone surfaces by Breton Tecnology. 2015. [acesso em 20 jul. 2015]. Disponível em: http://www.breton.it/dynamic/en/azienda/filosofia.php?PHPSESSID=7589075f46902 f6787a285ee1fd4a116.

4 Caesarstone. CaesarStone quartz surfaces. [acesso em 18 set. 2015]. Disponível em < http://www.caesarstoneus.com/catalog/technical-specs.cfm>.

5 Woodworking Network. US demand for cast polymers to reach 251 Million Sq. Ft. in 2016. [acesso em 12 jun. 2016]. Disponível em: http://www.woodworkingnetwork.com/wood-markettrends/woodworkingindustry-trendspress-releases/US-Demand-for-Cast-Polymers-to-Reach-251-Million-Sq-Ft-in-2016149739755.html

6 Associação Brasileira de Normas Técnicas. Solos - Análise granulométrica NBR 7181. Rio de Janeiro: ABNT; 1984.

7 Cornell JA. Experiments with mixtures: designs, models, and the analysis of mixture data. 3rd ed. New York: John Wiley \& Sons, 2002.

8 Associação Brasileira de Normas Técnicas. Solo - Determinação do índice de vazios mínimo de solos não-coesivos MB 3388 . Rio de Janeiro: ABNT, 1991.

9 Associação Brasileira de Normas Técnicas. Rochas para revestimento - métodos de ensaio NBR 15.584. Rio de Janeiro: ABNT, 2010.

10 Associación Española de Normalización y Certificación. Piedra aglomerada: Determinación de la resistencia a flexión 14617-2. Madrid: UNE-EN, 2008.

11 Associación Española de Normalización y Certificación. Piedra aglomerada: Determinación de la resistencia a compresión 14617-15. Madrid: UNE-EN, 2006.

12 Alicante. Aglostone: mármore composto. [acesso em 20 dez. 2015]. Disponível em: http://www.alicante.com.br/aglostone-2/.

13 Lee MY, Ko CH, Chang FC, Lo SL, Lin JD, Shan MY, Lee JC. Artificial stone slab production using waste glass, stone fragments and vacuum vibratory compaction. Cement \& Concrete Composites. 2008;30(7):583-587.

14 Chiodi Filho C, Rodriguez EP. Guia de aplicação de rochas em revestimentos. São Paulo: Abirochas, 2009.

15 American Society for Testing and Materials. Standard specification for marble dimension stone ASTM C 503. West Conshohocken: ASTM, 1999.

16 Carvalho EAS, Marques VR, Rodriguez RJS, Ribeiro CEG, Monteiro SN, Vieira CMF. Development of Epoxy Matrix Artificial Stone Incorporated with Sintering Residue from Steelmaking Industry. Materials Research, 2015;18(2):235-239.

17 Revestimentos de Mármore Composto. Ficha técnica de produto. [acesso em $04 \mathrm{dez}$. 2016]. Disponível em: http://eurosurfaces.eu/wp-content/uploads/2015/04/CremeLagoa_v01.pdf.

18 Borsellino C, Calabrese L, Di Bella G. Effects of powder concentration and type of resin on the performance of marble composite structures. Construction \& Building Materials, $2009 ; 23: 1915-1921$. 
19 Miller JD, Ishida H, Maurer FHJ. Dynamic-mechanical properties of interfacially modified glass sphere polyethylene. Rheologica Acta, 1998;27:397.

20 Debnath S, Ranade R, Wunder SL, Mccool J, Boberick K, Baran G. Interface effects on mechanical properties of particle-reinforced composites. Dent Mater, 2004;20(7): 67786.

21 Luz AB, Lins FF. Rochas e minerais industriais - Usos e especificacões. Rio de Janeiro: CETEM - MCT, 2005. 